\title{
Development of a Clinical Algorithm for the Early Diagnosis of Mucopolysaccharidosis III
}

Journal of Inborn Errors of Metabolism \& Screening 2020, Volume 8: e20200002 DOI: 10.1590/2326-4594-JIEMS-2020-0002

\author{
Maria Escolar ${ }^{1}$, Jessica Bradshaw², Valerie Tharp Byers ${ }^{3,}{ }^{*}$, Roberto Giugliani, ${ }^{4,5}$, Lynn Golightly', Charles \\ Marques Lourenço ${ }^{6}$ (1), Kimberly McDonald 7 , Nicole Muschol ${ }^{8}$, Imogen Newsom-Davis', Cara O’Neill ${ }^{3, *}$, \\ Holly L. Peay ${ }^{10}$, Jennifer Siedman ${ }^{11, *}$, Martha L. Solano ${ }^{12}$, Tessa Wirt ${ }^{1}$, Tim Wood ${ }^{13}$, Lonnie Zwaigenbaum ${ }^{14}$
}

\begin{abstract}
Mucopolysaccharidosis III (MPS III) is a rare inherited metabolic disease primarily affecting the central nervous system, leading to developmental and/or speech regression. Early diagnosis of the disease is important to introduce appropriate management measures and to optimize therapeutic outcomes. The diagnosis of MPS III is often significantly delayed due to the rarity of the disease, the more attenuated somatic presentation compared to other MPS types, and the symptom overlap with other developmental disorders. To shorten the time to diagnosis, a list of eight early signs and symptoms was identified through an expert system approach by a global, multidisciplinary working group of 13 specialists with expertise in various aspects of MPS and developmental disorders and three parents of MPS III patients. Coarse facial features and persistent hirsutism or prominent, thick eyebrows were identified as the most important MPS III early signs. The list of eight early MPS III signs and symptoms is the first step towards the development of a clinical algorithm aiming to identify neonates and infants with MPS III before the onset of neurocognitive damage, ultimately shortening the diagnostic journey of MPS III patients.
\end{abstract}

\section{Keywords:}

Mucopolysaccharidosis III, Sanfilippo syndrome, signs, symptoms, diagnostic algorithm.

\section{Background}

Mucopolysaccharidoses (MPSs) are a group of rare, autosomal recessive lysosomal storage disorders. Each MPS type is characterized by the absence or deficiency of a lysosomal enzyme involved in the catabolic pathway of glycosaminoglycans (GAGs), resulting in multi-organ accumulation of different GAGs and subsequent cellular damage[1-3]. MPS IV and VI manifest as purely somatic diseases with normal cognitive development[3]. The somatic manifestations in MPS IV and VI are similar to those seen in MPS I, II, and VII. However, the severe forms of the latter MPS types have both somatic and cognitive involvement [3]. Somatic features are relatively mild in MPS III or Sanfilippo syndrome, which is mainly characterized by cognitive and neurological impairment due to accumulation of the GAG heparan sulfate[2,4]. MPS III comprises four different subtypes: MPS IIIA, B, C, and D, which are the result from a deficiency in $\mathrm{N}$-sulfoglucosamine sulfohydrolase, alpha-N-acetylglucosaminidase, heparan acetyl-CoA:alpha-
1 UPMC Children's Hospital of Pittsburgh, Pittsburgh, PA, USA.

2 University of South Carolina, Department of Psychology, Columbia, SC, USA. ${ }^{3}$ Cure Sanfilippo Foundation, Columbia, SC, USA.

${ }^{4}$ Universidade Federal do Rio Grande do Sul, Hospital de Clínicas de Porto Alegre, Departamento de Genética, Porto Alegre, RS, Brasil.

${ }^{5}$ Instituto Nacional de Ciência e Tecnologia de Genética Médica Populacional (INAGEMP), Porto Alegre, RS, Brasil.

${ }^{6}$ Centro Universitário Estácio de Ribeirão Preto, Escola de Medicina, Ribeirão Preto, SP, Brasil.

${ }^{7}$ University of Mississippi Medical Center, Jackson, MS, USA.

${ }^{8}$ University Medical Center Hamburg-Eppendorf, International Center for Lysosomal Disorders (ICLD), Hamburg, Germany.

${ }^{9}$ Great Ormond Street Hospital NHS Foundation Trust, London, United Kingdom.

${ }^{10}$ RTI International, Center for Newborn Screening, Ethics, and Disability Studies, Durham, NC, USA.

${ }^{11}$ Autonomous Collaborator, Wellesley, MA, USA

${ }^{12}$ Fundación Cardio-Infantil; Bogota, Colombia

${ }^{13}$ Greenwood Genetics Center, Biochemical Genetics Laboratory, Greenwood, SC, USA.

${ }^{14}$ University of Alberta, Department of Pediatrics, Edmonton, AB, Canada.

Received February 27, 2020, and in revised form April 23, 2020. Accepted for publication April 28, 2020.

\section{Corresponding Author:}

Maria Escolar, UPMC Children's Hospital of Pittsburgh, Pittsburgh, PA, USA. Email: maria.escolar@chp.edu.

*Parents of MPS III patients. 
glucosaminide $\mathrm{N}$-acetyltransferase, and $\mathrm{N}$-acetylglucosamine6-sulfatase, respectively[1]. MPS III is one of the most common types of MPS with an estimated incidence ranging from 0.17 to 2.35 per 100,000 births for all four subtypes, with subtypes A and $\mathrm{B}$ being more frequently diagnosed than types $\mathrm{C}$ and $\mathrm{D}[5]$. Heparan sulfate mainly accumulates in the central nervous system, leading to early-onset neurodevelopmental and/or speech delay after an initial period of normal development[2]. Natural history studies have demonstrated that the first phase generally presents as a delay in speech and cognitive development occurring between the ages of 1 and 3 years for rapidly progressing phenotypes and around the age of 4 years for more slowly progressing phenotypes [2,4]. In rapidly progressing phenotypes, this first phase is immediately followed by a period of progressive neurocognitive decline, emergence of behavioral difficulties, and sleep disturbances. In slowly progressing phenotypes, especially in MPS IIIC and MPS IIID, the initial mild cognitive impairment may remain stable until the teenage years or even adulthood[6-8]. Somatic features such as coarse facial features, hepatomegaly, and umbilical hernia might appear throughout the disease course but are usually less pronounced than in other types of MPS[2,4]. During the third and final phase of the disease, motor function begins to decline and patients eventually progress to a vegetative state. MPS III patients have a poor prognosis, with life expectancy varying between the second and third decade of life[9]. However, life expectancy can range between the fourth and fifth decade for the slowly progressing phenotype in MPS IIIB[2]. Currently, no specific treatment option for MPS III exists, but several therapeutics are in clinical development $[10,11]$. These therapeutics are expected to result in optimal patient outcomes when initiated before neurological damage has occurred. This is supported by findings in MPS I, where hematopoietic stem cell transplantation can preserve cognitive development when performed before the onset of severe developmental deterioration[12,13]. Hence, it is becoming increasingly important to identify patients with MPS III as early as possible.

\section{Diagnostic Delay of MPS III}

Despite the importance of early diagnosis, diagnostic delays of more than 2 years are not uncommon in the MPS III population[4,14-16]. Studies, mainly including MPS IIIA patients, have shown that in $40.0 \%$ to $67.7 \%$ of children, initial symptoms appear before the age of 12 months. Common initial signs were sleep disturbances, behavioral abnormalities, speech delay, dysmorphology, and hearing loss[14,17]. Nevertheless, recent data from a study conducted in The Netherlands demonstrated that the median age at diagnosis remains 62 months (range 20-522 months). The diagnostic age was dependent on the disease phenotype, with rapidly progressing patients being diagnosed at a significantly younger age (mean age 54 months) than slowly progressing patients (mean age 71 months). In addition, the diagnostic delay, defined as the time between the first medical specialist visit and final diagnosis of MPS III, has not changed over the last 30 years (1988-2017). Although rapidly progressing patients were diagnosed earlier than slowly progressing patients, the median diagnostic delay was 33 months for both phenotypes[15]. The diagnostic delay may be due to poor disease awareness caused by the rarity of the disease, the absence or mild presentation of somatic symptoms, and the symptoms of the neurological progression (e.g., speech delay and behavioral problems) being similar to commonly seen developmental problems in the general population[15]. Furthermore, MPS III is not included in newborn screening programs and is often misdiagnosed as other developmental disorders such as idiopathic developmental delay, attention deficit hyperactivity disorder (ADHD), or autism spectrum disorder[2,18,19].

Besides primary care physicians and pediatricians, metabolic geneticists, orthopedic surgeons, ear-nose-throat specialists, audiologists, pediatric neurologists, and developmental specialists may be involved in the diagnostic journey of MPS III patients[2]. Although campaigns and initiatives to create more disease awareness among physicians and parents have been launched, reducing the diagnostic delay of MPS III remains a major challenge[20,21].

\section{Workshop Objective and Approach}

Here, we present the findings from a workshop held in December 2018 attended by an international multidisciplinary group of 13 specialists and three parents of MPS III patients. The group of specialists consisted of four specialists with extensive expertise in MPS, including three experts in (neurodevelopmental) pediatrics and one expert in clinical genetics and neurogenetics, two geneticists/lab directors, and seven specialists with expertise in clinical psychology, autism, speech pathology, clinical audiology, pediatric neurology, developmental pediatrics, and social sciences/bioethics. A panel discussion with the latter group of specialists aimed to identify signs and symptoms that could distinguish MPS III from other developmental disorders. In addition, the importance of including early parental concerns in the clinical setting was discussed. Following the discussions, an expert system approach[22] was applied to select the most important early signs and symptoms that should be tested in an MPS III early diagnosis algorithm aiming at reducing the average diagnostic age of MPS III patients to less than 2 years. For this purpose, the medical history, developmental milestones, and diagnostic pathways of different MPS III clinical cases were scored by the experts and parents on a 5-point scale according to the degree of suspicion for MPS III. The most important early signs and symptoms that should drive further testing for MPS III were defined by consensus generation and ranked according to importance based on the scoring of the clinical cases and 
clinical experience. Afterwards, the identified early signs and symptoms were supported by existing literature, if available.

\section{Workshop Outcome}

\section{Inclusion of Parental Concerns Into the Development of an MPS III Diagnostic Algorithm}

To accelerate diagnosis of MPS III, it is crucial to identify early concerns raised by parents and to establish which hurdles they might have in communicating these effectively to a healthcare provider. There is evidence that parental concerns about their child's development are predictive of later developmental deficits in more than $80 \%$ of the cases[23]. Studies in the autism field have demonstrated that parents often express concerns before receiving a diagnosis, emphasizing the need for healthcare providers to seriously elicit parental concerns $[24,25]$.

A major challenge with parental concerns is that they are often diffuse and difficult for healthcare providers to pursue. An example of an initial concern of parents of children diagnosed with MPS III was "he has a lot of thick hair", which in hindsight might have been related to hirsutism. How inclusion of parental concerns in the diagnostic pathway can be promoted is illustrated in the field of muscle weakness disorders, including Duchenne and Becker, congenital muscular dystrophies, and spinal muscular atrophy[26]. To prompt early diagnosis of these diseases, pre-diagnostic experiences of parents presenting their earliest concerns to their healthcare providers are described on a website, ChildMuscleWeakness.org[26]. Reliable patient websites can be extremely helpful for parents as an initial source of information and can facilitate early diagnosis by including the parental voice.

Another challenge with parent-reported data is retrospective bias due to incorrect recall of signs and symptoms. Electronic medical records may also not adequately document early signs and symptoms reported by parents, and therefore do not always allow a robust assessment of the early developmental trajectory. In addition, parents might not be willing to seek a diagnosis and may downplay initial (unspecific) signs because they are unable to cope with their child's symptoms, particularly early in the disease course. Education of parents, caregivers, and physicians on early signs and symptoms of MPS III can help to distinguish "normal" from "clinically significant" parental concerns.

Furthermore, experience with the ChildMuscleWeakness. org program revealed that the broadest reasonable range of possible disorders should be included under a parent-reported assessment due to overlap of early signs with other conditions, the rarity of each diagnosis, and the increased ability to capture primary care provider attention with education about a related set of disorders with a much higher prevalence. More specifically, differential diagnosis of MPS III should be part of the diagnostic algorithms of other developmental disorders such as ADHD, hearing loss, and autism spectrum disorder. By implementing
MPS III signs and symptoms into the differential diagnosis of other developmental disorders, providers are more likely to consider MPS III in their differential diagnoses.

\section{Early MPS III Signs and Symptoms Before the Onset of Developmental Delay}

In line with the goal of diagnosing neonates and infants before the onset of neurological damage, Table 1 summarizes a list of early signs and symptoms, identified during the workshop, that might become apparent before the age of 1 year. These signs and symptoms are believed to warrant referral in a timely manner and to shorten the diagnostic trajectory of MPS III patients.

Some of these early signs and symptoms, such as diarrhea, are rather nonspecific and do not facilitate differential diagnosis, while other symptoms such as coarse facial features, persistent hirsutism and synophrys, accelerated growth during the $1^{\text {st }}$ year of life, and macrocephaly are more characteristic of MPS III $[1,14,27]$. Part of the signs and symptoms that were identified, including behavioral problems, speech regression, coarse facial features, gastro-intestinal problems, mouthing, sensorineural hearing loss, hip dysplasia, and sleep disturbances, might only occur after the age of 1 year, especially in patients with a more slowly progressing disease.

Behavioral problems and speech regression often resemble ADHD-like behaviors or autism respectively, resulting in delayed diagnosis of MPS III due to misdiagnosis, which can be even more delayed when symptomatic treatment initially provides some relief of these symptoms[2,28]. However, subtle behavioral differences supporting the differential diagnosis do exist and the autistic-like symptoms of MPS III patients differ from those seen in children with autism spectrum disorder in several ways. One subtle difference that might distinguish MPS III from autism spectrum disorder is the initial period of normal development followed by the acquisition of autism symptoms during first 3 to 4 years of life in patients with MPS III[28]. This regression has also been reported in $16 \%$ to $50 \%$ of patients with autism spectrum disorder but appears to occur earlier, between the first and second birthday[29]. Furthermore, MPS III children might preserve functional play skills while the play behavior of children with autism spectrum disorder can be more limited or non-functional and characterized by repetitive and restricted patterns. In addition, unlike many children with autism spectrum disorder, MPS III patients might initially develop robust joint attention skills, enjoy engaging in social interaction without anxiety, and easily adjust to changes in routine. The increase in anxiety seen in children with MPS III coincides with the speech regression and the inability to communicate and express themselves, which can lead to frustration and aggressive behavior. Although autistic-like behaviors do exist in some MPS III children, the most common and typical restrictive and repetitive behavior present in children with MPS III is mouthing or oral fixation. The absence of other restrictive and repetitive behaviors might differentiate MPS III 
Table 1. Early signs and symptoms of MPS III patients identified by the experts and parents through the expert system approach. The experts' observations were supported by existing medical literature, if available.

\begin{tabular}{|c|c|}
\hline Early sign/symptom & Expert comments and/or parental concerns and supporting medical literature \\
\hline Hearing loss & $\begin{array}{l}\text { Expert comments and/or parental concerns } \\
\text { - Most MPS III patients do not fail the hearing test on newborn screening. Therefore, the hearing test on newborn screening is not } \\
\text { always a reliable predictor. } \\
\text { - Children with another cause of hearing loss are generally more engaged and attempt to compensate for hearing loss by actively } \\
\text { trying to find other signals, although lack of engagement might also be related to developmental disorders other than MPS III. } \\
\text { - An auditory brainstem response (ABR) could be informative. An ABR can demonstrate prolonged latencies in MPS III patients } \\
\text { compared to the latencies of others with similar configurations of hearing loss. Typically, ABRs are coordinated with other } \\
\text { procedures for which the patient is already under anesthesia. Tympanometry is usually conducted in conjunction with ABRs. } \\
\text { - Although hearing loss in MPS III is typically sensorineural, conductive components might be present due to recurring ear } \\
\text { infections or middle ear disease which might mask mild sensorineural hearing loss. Sensorineural hearing loss typically starts as a } \\
\text { mild, sloping high-frequency hearing loss that progresses over time. } \\
\text { Supporting medical literature } \\
\text { - The type of hearing loss, conductive versus sensorineural, can give clues regarding the potential presence of MPS III, which is } \\
\text { typically characterized by sensorineural hearing loss and altered ABRs[14,31]. }\end{array}$ \\
\hline Craniosynostosis & $\begin{array}{l}\text { Expert comments and/or parental concerns } \\
\text { - Craniosynostosis was identified by the experts as potential early symptom, although it is not a common presentation in MPS III in } \\
\text { comparison to MPS I, II, and VI. } \\
\text { Supporting medical literature } \\
\text { - Craniosynostosis affects the majority of MPS patients regardless of the MPS type[32]. }\end{array}$ \\
\hline
\end{tabular}

Expert comments and/or parental concerns

- An important parental concern was sleep disturbance, such as night time anxiety and abnormal nocturnal activity. Parents and experts perceive sleeping problems to be worse in MPS IIIA than in MPS IIIB patients.

Sleep disturbance Supporting medical literature

- Fraser et al. showed that $91.5 \%$ of children with MPS III suffer from sleep disturbance (age of onset: birth to 22 years). Sleep disturbance occurs more frequently in MPS III patients versus their unaffected siblings[8].

Expert comments and/or parental concerns

- Early respiratory problems: upper respiratory congestion of nose and/or ears with mucus production and neonatal breathing difficulties.

ENT problems

- Late respiratory problems: aspiration pneumonia.

Supporting medical literature

- Evidence confirms the presence of chronic sinusitis and otitis media[4,14,33].

Expert comments and/or parental concerns

- Children with MPS III tend to overeat and have an abnormal focus on food, repeated colic-like episodes, and recurrent diarrhea.

Gastrointestinal Supporting medical literature

problems

- In a natural history cohort of Spanish MPS III patients, recurrent diarrhea was present in 50\% of cases before diagnosis[4].

- There is no medical literature supportive of the tendency to overeat in MPS III patients. However, MPS IIIA mice exhibited greater bodyweight gain than controls, which was explained by abnormalities in gastrointestinal structure and function. Whether these findings translate to humans is unclear[34].

Expert comments and/or parental concerns

- Umbilical and/or inguinal hernias frequently occur and are thought to be less responsive to surgery in MPS III patients versus healthy subjects.

Umbilical or inguinal Supporting medical literature

hernia

- Umbilical hernia and inguinal hernia were reported in $22 \%$ and $7 \%$ of MPS III patients at a median age of 1 month (range 0-8 months) and 1.6 years (range 0-3 years), respectively[4]. However, umbilical hernias frequently occur in newborns affecting an estimated $10-30 \%$ of all white children at birth and an estimated $23-85 \%$ of children in the African population, while inguinal hernias occur in $0.8 \%$ to $5 \%$ of infants[35].

Expert comments and/or parental concerns

- Mostly parents noticed the presence of persistent and excessive thick body hair, prominent eyebrows, and synophrys.

Hirsutism Supporting medical literature

- Hirsutism was reported as the most prevalent physical symptom (93\%) and the second most common initial sign (64\%) in a Taiwanese cohort of MPS III patients[36]. 
Table 1. Cont.

Early sign/symptom

Expert comments and/or parental concerns and supporting medical literature

Expert comments and/or parental concerns

- Frontal bossing, coarse facial features, puffiness underneath the eyes and eyebrows, and a particular lip morphology with a narrow upper lip and a thick lower lip were identified.

Facial abnormalities

- Macrocephaly, especially when presented as early as at birth.

Supporting medical literature

- Coarse facial features were present before diagnosis in 78\% of MPS III patients in a Spanish natural history cohort[4].

- Head circumference is increased within the first 2 years of life until physical maturity and is followed by a gradual decline likely related to the progressive loss of brain tissue[14,17,27,37]

Expert comments and/or parental concerns

- Physically, MPS III patients might appear older than their actual age, but because of their lower intellectual ability, hyperactivity, and balance issues, their physical and intellectual maturity is often incongruent.

Abnormal growth Supporting medical literature

- No normal Gaussian distribution of the growth curve. MPS III patients are in the highest percentile of the growth curve[14,37].

- A recent study developed growth charts of German MPS III patients. MPS III patients showed an accelerated growth during the $1^{\text {st }}$ year of life but a decelerated growth rate after 4.5 and 5 years for females and males, respectively[27].

Expert comments and/or parental concerns

- Although speech only develops around the age of 12 months, patients with MPS III might already have subtle impairments in communication at a younger age. More in-depth analysis may reveal MPS III-specific communication profiles in infants.

- Joint attention (the infant's ability to join the focus of attention of other persons)[38] and the ability to engage with the environment might initially be better developed in MPS III compared with autism spectrum disorder (personal communication).

Speech and MPS III patients might initially be more engaged with their parents and the environment and make more eye contact than autism patients. These differences might be helpful in the differential diagnosis.

- The ability to speak is gradually lost while motor development is preserved longer. Supporting medical literature

- Studies demonstrated onset of speech regression at the age of 2.8 years (SD: 1.9) and regression of motor function at 4.1 years (SD: 3.6) for MPS IIIA and similar regression profiles for MPS IIIB[17].

- Speech delay with an initial period of normal motor development might lead to misdiagnosis of an idiopathic speech delay[2,4]. Expert comments and/or parental concerns

- Patients with MPS III can have impulsive and fearless behavior. Fearless behavior is difficult to interpret below the age of 2 years. Parents usually only report fearless and impulsive behavior when this becomes abnormal.

- Hypersensitivity and attention problems frequently occur and can manifest as early as 12-24 months or appear at later ages. MPS III patients typically present autistic-like behavior. Features that might differentiate MPS III from autism are appropriate and functional play, longer attention span and more engagement, fearlessness, less impaired social skills, usually less repetitive or restricted behavior, and no ritualistic behaviors. Children with MPS III also appear to be more aggressive, likely associated with

Behavioral problems their inability to express themselves due to speech delay and hearing loss.

Supporting medical literature

- An increased incidence of autistic-like social behaviors occurred between 3-4 years of age in a cohort of children with early-onset MPS IIIA[28].

- An increased incidence of behavioral problems emerges between the age of 3 and 4 years and presents as a decrease in engagement with the environment[4,28]

- Hyperactive and fearless behavior that does not respond to standard of care, such as pharmacotherapy for ADHD, differentiating both disorders[2].

Expert comments and/or parental concerns

- Hip dysplasia initially presents as hip inflexibility, reported by the parents as the inability to sit cross-legged.

Hip dysplasia Supporting medical literature

- Musculoskeletal symptoms are less pronounced in MPS III versus other types of MPS, but hip deformities are among the most common orthopedic abnormalities and are related to disease severity[39,40].

Expert comments and/or parental concerns

Pain sensation

- Based on the participants' experiences, MPS III patients tend to have increased pain tolerance, e.g., MPS III children often do not flinch when blood is drawn or do not feel pain when falling down. However, currently no supporting evidence can be found, and further studies would need to examine if MPS III children experience and/or express pain differently.

Expert comments and/or parental concerns

- Parents and experts reported mouthing in various cases of MPS III.

Mouthing Supporting medical literature

- Restrictive and repetitive behaviors such as hand flapping or rocking are mostly absent while oral restricted behaviors and hyperorality can be present[28]. 
from autism spectrum disorder[28]. Early presentation of macrocephaly in children with MPS III, sometimes as early as birth, might further distinguish both disorders[14]. Another MPS III-specific, albeit later sign, is the regression of speech, which usually precedes the deterioration of motor function[17]. The trajectory of speech regression appears to be specific for MPS III and is characterized by a gradual loss of language. In addition, hearing loss is often observed early on in MPS III while being absent in autism spectrum disorder[14].

As most signs and symptoms summarized in Table 1 are not exclusively presenting before the age of 1 year, consensus was reached on a final list of eight early presenting signs and symptoms in neonates and infants dependent on the likelihood that the particular signs or symptoms are MPS III-specific (Table 2). Each sign or symptom was attributed a score ranging from 1 to 3 . A score of 1 corresponds to a rather unspecific sign while a score of 3 is considered to be highly characteristic of MPS III. Coarse facial features and persistent hirsutism or prominent thick eyebrows, which have previously been reported as MPS III-specific signs, should prompt referral to a metabolic physician and/or developmental specialist. Presence of both facial coarsening and prominent thick eyebrows before the age of 2 is illustrated in Figure 1, showing the progression of MPS III patients in comparison with their unaffected siblings. Breathing problems within the first week of life, frontal bossing and macrocephaly (i.e. head circumference above the $99^{\text {th }}$ percentile), and an increased need for nursing were classified as having only some specificity for MPS III. Lastly, colic-like episodes and gastrointestinal discomfort, umbilical or inguinal hernia, and upper respiratory congestion are considered to be prevalent among neonates and infants. Screening for this combination of unspecific and specific signs and symptoms is anticipated to raise suspicion for MPS III as early as possible and to shorten the diagnostic journey of MPS III patients.

Table 2. Final list of early MPS III signs and symptoms.

\begin{tabular}{lc}
\hline Sign/symptom & Score* \\
\hline Coarse facial features & 3 \\
Persistent hirsutism or prominent thick eyebrows & 3 \\
Breathing problems (1 ${ }^{\text {st }}$ week of life) & 2 \\
Frontal bossing and macrocephaly & 2 \\
Increased need for nursing and food intake & 2 \\
Colic-like episodes and gastrointestinal discomfort & 1 \\
Umbilical or inguinal hernia & 1 \\
Upper respiratory congestion & 1 \\
\hline
\end{tabular}

*A score of 1 corresponds to a rather unspecific sign while a score of 3 is considered to be highly characteristic of MPS III. A total score of 9-15 would be highly suggestive of MPS III, a score of 3-9 would raise suspicion of MPS III, and a score of $0-3$ would not be suggestive of MPS III.

\section{Early Diagnosis Algorithm - Future Perspectives and Barriers to Overcome}

In order to validate the observations, the incidence of the early signs and symptoms summarized in Table 2 will be retrospectively and prospectively reviewed in clinical cases of MPS III, cases of developmental delay, and healthy controls. Some of the signs and symptoms might be eliminated when they fail to distinguish neonates and infants with MPS III from the healthy control group. Other signs and symptoms might be up-/down-rated when they are found to be more/less prevalent in young MPS III patients than initially anticipated. The ultimate goal is to develop an easy-to-use early diagnostic algorithm to be used mainly by primary care physicians and pediatricians as they are most likely to be consulted first by parents. Dependent on the MPS III suspicion score, follow-up, testing, and referral to a clinical geneticist or developmental specialist is recommended. Diagnostic testing for MPS III can be performed using a variety of samples depending on the available resources. Testing can include screening methods such as analysis of urinary GAGs, enzymatic analysis in blood or cultured fibroblasts, or genetic analysis of MPS III genes. Depending on the testing ordered, results are typically available within several weeks or a month[30].

Further recommendations of the multidisciplinary group to increase disease awareness among physicians not familiar with MPS III were to increase awareness of facial dysmorphisms and MPS III-like behaviors through videos and pictures. In addition, physicians should try to elicit parental concerns about their child's development. It is expected that interest in MPS III and possibly newborn screening will increase once treatment options are available. However, until then, an early diagnosis algorithm could already be implemented into the differential diagnosis of other neurodevelopmental or genetic disorders, such as autism spectrum disorder.

Besides challenges associated with creating disease awareness and implementation of an early diagnosis algorithm, shortening referral times in healthcare will be a major hurdle for improving early diagnosis of MPS III. Waiting times between the first medical visit and an appointment with a metabolic geneticist or neurodevelopmental specialist can be as long as 18 months as recently determined in a cohort of Dutch MPS III patients[15]. Therefore, primary care physicians and pediatricians should consider an accelerated referral after profound suspicion of MPS III based on the early diagnosis algorithm. Ultimately, newborn screening will be needed to diagnose MPS III before symptoms, but until this is available, early diagnosis is necessary for optimal outcomes of novel therapies.

\section{Conclusion}

MPS III is a rare inherited metabolic disease with poor prognostic outcomes due to severe neurological deficits and the absence of 
A

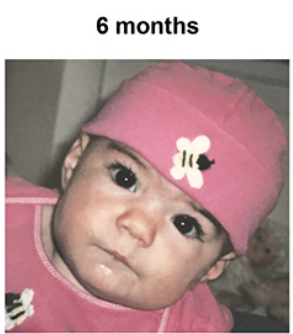

7 months

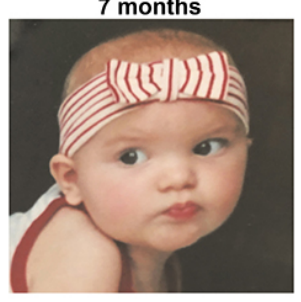

24 months

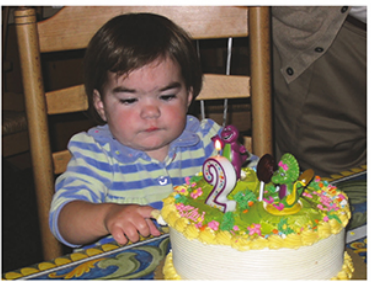

22 months

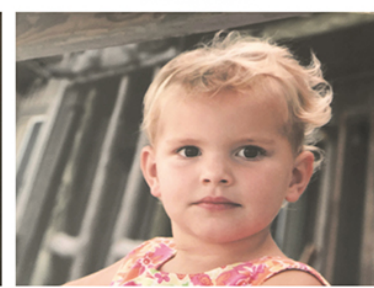

4.5 years (left) -2.5 years (right)

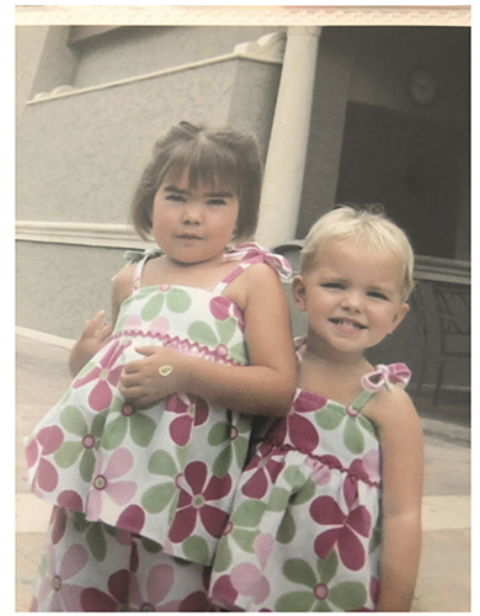

B

14 months
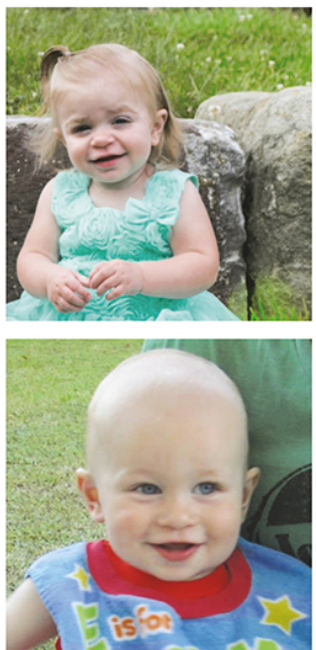

24 months


37-38 months
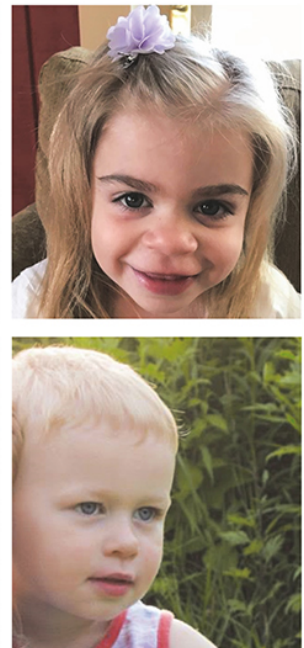

C
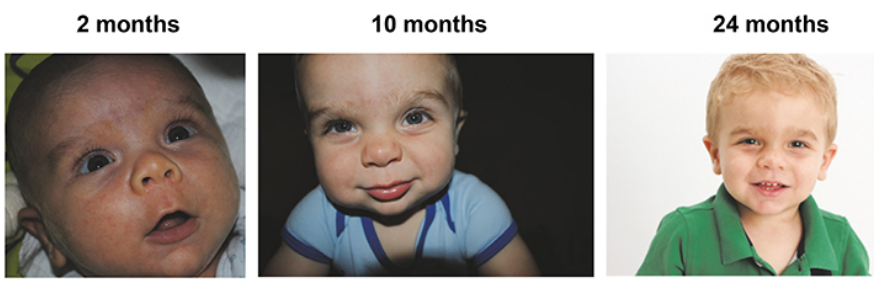

5 years
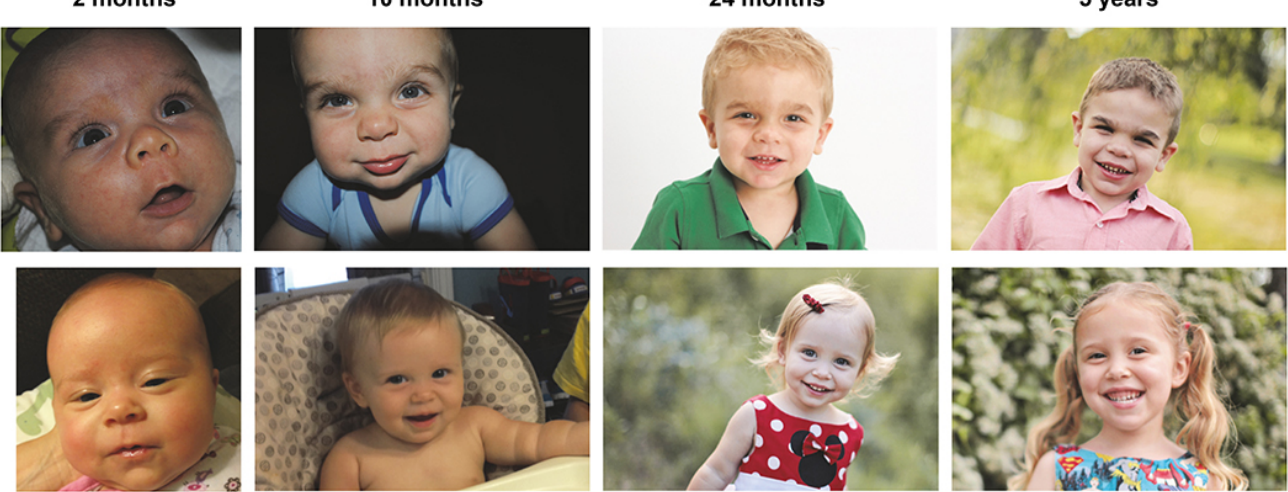

Figure 1. Early facial coarsening and presence of synophrys in MPS IIIB patients. (A) Left: progression of an MPS IIIB patient (upper panel) in comparison with her unaffected sibling (lower panel) at comparable ages. Right: comparison of the MPS IIIB patient (left) with her unaffected sibling (right) at different ages. (B \& C) Progression of two MPS IIIB patients (upper panels) in comparison with their age-matched unaffected siblings (lower panels). 
curative treatment options. Although new treatment options for the disease are being developed, neurocognitive deterioration will likely only be prevented if treatment is initiated before the onset of developmental delay. The consensus list of early signs and symptoms presented here is a crucial step towards the development of an early diagnosis algorithm for patients with MPS III. This list was developed based on current evidence and experiences from a multidisciplinary group of MPS experts and MPS III parents. These observations can be retrospectively and prospectively validated with more in-depth analysis of the early development of MPS III patients. An early diagnostic algorithm developed based on these early signs and symptoms is expected to eventually shorten the diagnostic journey of MPS III patients until newborn screening is available for this disorder.

\section{Abbreviations}

ABR: auditory brainstem response; ADHD: attention deficit hyperactivity disorder.; ENT: ear-nose-throat; GAGs: glycosaminoglycans; MPS: mucopolysaccharidosis.

\section{Acknowledgements}

The authors are grateful to Ismar Healthcare NV who provided medical writing assistance, which was funded by BioMarin Pharmaceutical Inc.

\section{Funding}

This work was supported by BioMarin Pharmaceutical Inc. and Allievex.

\section{Ethics and Consent}

Consent was obtained to publish the patients' pictures.

\section{Authors' Contributions}

All authors were actively involved in the workshop on which the publication is based. All authors read and approved the final manuscript.

\section{Declaration of Conflict of Interests}

The content of this manuscript is based on discussions during a workshop meeting that was coordinated and funded by BioMarin Pharmaceutical Inc. All authors received funding to participate in this meeting. CML has received honoraria for speaker's fees from Actelion, Genzyme, BioMarin, PTC, and Shire HGT; all fees are donated to the CML Foundation for Research and Genetic Diagnosis Support for families with unknown genetic disorders. ME is a consultant for BioMarin and Abeona Therapeutics. NM is a consultant for BioMarin and has received grants/research support as well as honoraria and travel grants from BioMarin. Although unrelated to this work, RG received compensation for activities as investigator, and/or consultant, and/or speaker for Abeona, Actelion, Amicus, Armagen, BioMarin, CentoGene, GC Pharma, Inventiva, JCR Pharmaceuticals, Lysogene, Protalix, PTC, RegenxBio, Sanofi Genzyme, Shire, Sobi, and Ultragenyx.

\section{References}

1. Andrade F, Aldámiz-Echevarría L, Llarena M and Couce ML. Sanfilippo syndrome: Overall review. Pediatr Int. 2015; 57(3):331-338. doi:10.1111/ped.12636

2. Wijburg FA, Wegrzyn G, Burton BK and Tylki-Szymanska A. Mucopolysaccharidosis type III (Sanfilippo syndrome) and misdiagnosis of idiopathic developmental delay, attention deficit/hyperactivity disorder or autism spectrum disorder. Acta Paediatr. 2013;102(5):462-470. doi:10.1111/ apa.12169

3. Muenzer J. Overview of the mucopolysaccharidoses. Rheumatology (Oxford). 2011;50(Suppl 5): v4-v12. doi:10.1093/rheumatology/ker394

4. Delgadillo V, O’Callaghan MDM, Gort L, Coll MJ and Pineda M. Natural history of Sanfilippo syndrome in Spain. Orphanet J Rare Dis. 2013; 8:189. doi:10.1186/1750-11728-189

5. Zelei T, Csetneki K, Voko Z and Siffel C. Epidemiology of Sanfilippo syndrome: results of a systematic literature review. Orphanet J Rare Dis. 2018; 13(1):53. doi:10.1186/ s13023-018-0796-4

6. Valstar MJ, Marchal JP, Grootenhuis M, Colland V and Wijburg FA. Cognitive development in patients with Mucopolysaccharidosis type III (Sanfilippo syndrome). Orphanet J Rare Dis. 2011;6: 43. doi: 10.1186/1750-11726-43

7. Bax MCO and Colville GA. Behaviour in mucopolysaccharide disorders. Arch Dis Child. 1995;73(1): 7781. doi: $10.1136 /$ adc.73.1.77

8. Fraser J, Gason AA, Wraith JE and Delatycki MB. Sleep disturbance in Sanfilippo syndrome: a parental questionnaire study. Arch Dis Child. 2005;90(12): 12391242. doi:10.1136/adc.2004.065482

9. Lavery C, Hendriksz CJ and Jones SA. Mortality in patients with Sanfilippo syndrome. Orphanet J Rare Dis. 2017;12: 168. doi:10.1186/s13023-017-0717-y

10. Aoyagi-Scharber M, Crippen-Harmon D, Lawrence R, et al. Clearance of heparan sulfate and attenuation of CNS pathology by intracerebroventricular BMN 250 in Sanfilippo type B mice. Mol Ther Methods Clin Dev. 2017; 6:43-53. doi: 10.1016/j.omtm.2017.05.009

11. Gilkes JA, Bloom $\mathrm{MD}$ and Heldermon CD. Mucopolysaccharidosis IIIB confers enhanced neonatal intracranial transduction by AAV 8 but not by 5,9 or rh 10 . Gene Ther. 2016;23(3):263-271. doi: 10.1038/gt.2015.111

12. Souillet G, Guffon N, Maire I, et al. Outcome of 27 patients with Hurler's syndrome transplanted from either related or unrelated haematopoietic stem cell sources. Bone Marrow Transplant. 2003;31(12): 1105-1117. doi:10.1038/ sj.bmt.1704105

13. Poe MD, Chagnon SL and Escolar ML. Early treatment is associated with improved cognition in Hurler syndrome. Ann Neurol. 2014;76(5):747-753. doi:10.1002/ana.24246 
14. Buhrman D, Thakkar K, Poe M and Escolar ML. Natural history of Sanfilippo syndrome type A. J Inherit Metab Dis. 2014;37(3):431-437. doi:10.1007/s10545-013-9661-8

15. Kuiper GA, Meijer OLM, Langereis EJ and Wijburg FA. Failure to shorten the diagnostic delay in two ultra-orphan diseases (mucopolysaccharidosis types I and III): potential causes and implications. Orphanet J Rare Dis. 2018;13:2. doi:10.1186/s13023-017-0733-y

16. Vieira T, Schwartz I, Muñoz V, et al. Mucopolysaccharidoses in Brazil: what happens from birth to biochemical diagnosis? Am J Med Genet A. 2008;146A(13):1741-1747. doi:10.1002/ ajmg.a.32320

17. Meyer A, Kossow K, Gal A, et al. Scoring evaluation of the natural course of mucopolysaccharidosis type IIIA (Sanfilippo syndrome type A). Pediatrics. 2007;120(5):e1255-e1261. doi:10.1542/peds.2007-0282

18. Wolfenden C, Wittkowski A, Jones SA, Rust S and Hare DJ. Autism spectrum disorder symptomatology in children with mucopolysaccharide disease type III. $\mathrm{Br}$ J Learn Disabil. 2019;47:5-11. doi:10.1111/bld.12248

19. Shapiro E, King K, Ahmed A, et al. The neurobehavioral phenotype in mucopolysaccharidosis type IIIB: an exploratory study. Mol Genet Metab Rep. 2016;6:41-47. doi:10.1016/j.ymgmr.2016.01.003

20. Cure Sanfilippo Foundation. https://curesff.org/

21. Sanfilippo Children's Foundation. https://www.sanfilippo. org.au/

22. Escolar ML, Poe MD, Martin HR and Kurtzberg J. A staging system for infantile Krabbe disease to predict outcome after unrelated umbilical cord blood transplantation. Pediatrics. 2006;118:e879-889. doi:10.1542/peds.2006-0747

23. Lurio JG, Peay HL and Mathews KD. Recognition and management of motor delay and muscle weakness in children. Am Fam Physician. 2015; 91(1): 38-44. https:// www.aafp.org/afp/2015/0101/p38.html

24. Richards M, Mossey J and Robins DL. Parents' concerns as they relate to their child's development and later diagnosis of autism spectrum disorder. J Dev Behav Pediatr. 2016;37(7):532-540. doi:10.1097/dbp.0000000000000339

25. Guinchat V, Chamak B, Bonniau B, et al. Very early signs of autism reported by parents include many concerns not specific to autism criteria. Res Autism Spectr Disord. 2012;6: 89-601. doi:10.1016/j.rasd.2011.10.005

26. National Task Force for Early Identification of Childhood Neuromuscular Disorders. Child Muscle Weakness. https:// www.childmuscleweakness.org/

27. Muschol NM, Pape D, Kossow K, et al. Growth charts for patients with Sanfilippo syndrome (Mucopolysaccharidosis type III). Orphanet J Rare Dis. 2019;14:93. doi:10.1186/ s13023-019-1065-x
28. Rumsey RK, Rudser K, Delaney K, Potegal M, Whitley $\mathrm{CB}$ and Shapiro E. Acquired autistic behaviors in children with mucopolysaccharidosis type IIIA. J Pediatr. 2014;164(5):1147-1151.e1. doi:10.1016/j.jpeds.2014.01.007

29. Ozonoff D, Heung K, Byrd R, et al. The onset of autism: patterns of symptom emergence in the first years of life. Autism Res. 2008;1(6):320-328. doi:10.1002/aur.53

30. Bodamer OA, Giugliani R, Wood T. The laboratory diagnosis of mucopolysaccharidosis III (Sanfilippo syndrome): A changing landscape. Mol Genet Metab. 2014;113(1-2):3441. doi:10.1016/j.ymgme.2014.07.013

31. Zanetti D, Vezzani M, Di Berardino F, et al. Characterization of hearing loss in children with mucopolysaccharidosis. An Excursus into Hearing Loss. London, UK: IntechOpen; 2018.

32. Oussoren E, Mathijssen IMJ, Wagenmakers M, et al. Craniosynostosis affects the majority of mucopolysaccharidosis patients and can contribute to increased intracranial pressure. J Inherit Metab Dis. 2018;41(6):12471258. doi:10.1007/s10545-018-0212-1

33. Yodoshi T and Hurt TL. Avoiding diagnostic delay for mucopolysaccharidosis IIIB: do not overlook common clues such as wheezing and otitis media. BMJ Case Rep. 2018;2018:bcr-2018-224412. doi:10.1136/bcr-2018-224412

34. Roberts AL, Howarth GS, Liaw WC, et al. Gastrointestinal pathology in a mouse model of mucopolysaccharidosis type IIIA. J Cell Physiol. 2009;219(2):259-64. doi:10.1002/ jcp. 21682

35. Bowling K, Hart N, Cox P and Srinivas G. Management of paediatric hernia. BMJ. 2017;359:j4484. doi:10.1136/ bmj.j4484

36. Lin HY, Chuang CK, Lee CL, et al. Mucopolysaccharidosis III in Taiwan: natural history, clinical and molecular characteristics of 28 patients diagnosed during a 21 year period. Am J Med Genet A. 2018;176(9): 1799-1809. doi:10.1002/ajmg.a.40351

37. de Ruijter J, Broere L, Mulder MF, et al. Growth in patients with mucopolysaccharidosis type III (Sanfilippo disease). $J$ Inherit Metab Dis. 2014;37(3):447-454. doi:10.1007/s10545013-9658-3

38. Akhtar $\mathrm{N}$ and Gernsbacher MA. Joint attention and vocabulary development: a critical look. Lang Linguist Compass. 2007;1(3):195-207. doi:10.1111/j.1749-818X. 2007.00014.x

39. White KK, Karol LA, White DR and Hale S. Musculoskeletal manifestations of Sanfilippo Syndrome (mucopolysaccharidosis type III). J Pediatr Orthop. 2011;31(5):594-598. doi:10.1097/bpo.0b013e31821f5ee9

40. de Ruijter J, Maas $M$, Janssen A and Wijburg FA. High prevalence of femoral head necrosis in Mucopolysaccharidosis type III (Sanfilippo disease): a national, observational, cross-sectional study. Mol Genet Metab.2013;109(1):49-53. doi:10.1016/j.ymgme.2013.03.004 